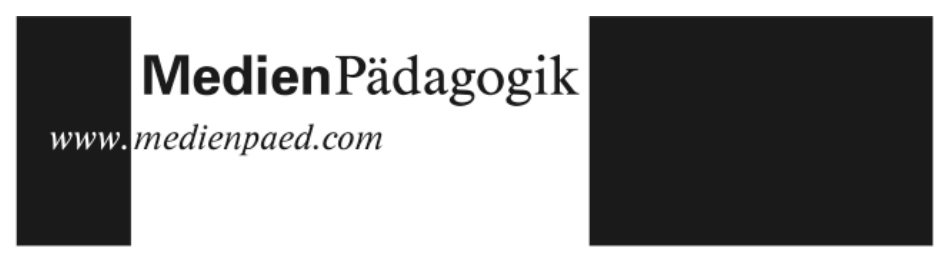

\title{
Rezensionen
}

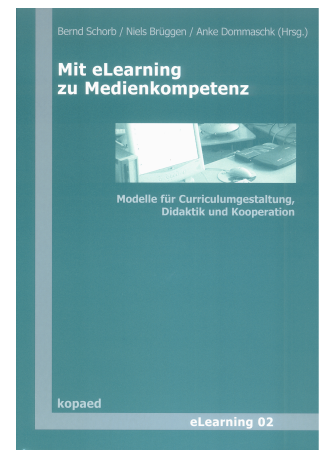

Bernd Schorb, Niels Brüggen u. Anke Dommaschk (Hrsg.)

Mit eLearning zu Medienkompetenz

Modelle für Curriculumgestaltung, Didaktik und Kooperation

München: kopaed, 2008. 224 Seiten

ISBN: 3-86736-000-6

$€ 16.80 ; \mathrm{CHF} 30,90$

\section{Mit eLearning zu Medienkompetenz?}

Der Band Mit eLearning zu Medienkompetenz beschäftigt sich mit Qualifizierungsmodellen zu Medienkompetenz im Bereich der universitären Aus- und Weiterbildung von Lehrern und Lehramtsstudierenden. Damit stellt der Band einen Beitrag zu drei hoch aktuellen Themen dar; zum einen zur wissenschaftlichen Weiterbildung als zentrale Aufgabe von Universitäten vor dem Hintergrund des Bologna-Prozesses. Zweitens zur Entwicklung von Curricula zur Vermittlung von Medienkompetenz, als eine entscheidende Kompetenz im Rahmen der Wissensgesellschaft; und schließlich zur didaktischen Gestaltung von eLearning-Szenarien, als effiziente und flexible Form der Umsetzung von Weiterbildungsangeboten.

Den Hintergrund des Bandes bilden die Erfahrungen aus einem von der BLK geförderten Modellprojekt «Verbund in der Weiterbildung für Medienkompetenz - Ein Online-Angebot sächsischer Hochschulen». Die Beiträge des Bandes beziehen sich zu einem Teil auf das Modellprojekt, weisen aber auch über dieses hinaus und greifen weiterführende Fragen aus dem Bereich der wissenschaftlichen Weiterbildung, des eLearning und der Vermittlung von Medienkompetenz auf. Die Schwerpunkte liegen dabei zum einen auf der inhaltlichen und organisatorischen Gestaltung eines wissenschaftlichen Aus- und Weiterbildungscurriculums im Bereich Medienkompetenzvermittlung und zum anderen auf bereits umgesetzten und erprobten Modellen mit einer ähnlichen thematischen Ausrichtung. Der Band gibt dabei Einblicke in die unterschiedlichen Bereiche und Ebenen, die von einem solchen Vorhaben betroffen sind. Der erste 
Teil widmet sich vornehmlich den Rahmenbedingungen, die für die Realisierung eines wissenschaftlichen Weiterbildungscurriculums relevant sind. Schorb entwickelt in seinem Beitrag, ausgehend von bestehenden Modellen, ein Verständnis von Medienkompetenz, das die Dimensionen Wissen, Bewerten und Handeln umfasst und diskutiert dessen Bezug zum Begriff Medienbildung. Damit wird zum einen die Diskussion über die Operationalisierung von Medienkompetenz angerissen, zum anderen eine Basis für die Entwicklung eines Medienkompetenzcurriculums formuliert. Spanhel konkretisiert ein solches Curriculum in seinem Beitrag weiter, indem er auf die medienpädagogische Qualifizierung von Lehrkräften eingeht, konkrete Inhalte benennt und ein didaktisches (blendedlearning) Konzept zu deren Vermittlung darstellt. Schäfer geht in seinem Beitrag auf die hochschulpolitischen Vorgaben ein, die aus dem Bologna-Prozess resultieren und erläutert, wie sich diese auf universitäre (weiter-)Bildungsangebote niederschlagen. Der Beitrag von Dommaschk zeigt am Beispiel von SOMEK (sächsisches Online-Bildungsangebot Medienkompetenz) wie die in den vorausgegangen Artikeln entfalteten Aspekte konkretisiert und in ein Curriculum zur Vermittlung von Medienkompetenz eingebunden werden können.

Der zweite Teil beschäftigt sich mit didaktischen und methodischen Fragen im Bereich der online-gestützten Lehre und Weiterbildung. Hier wird die Bedeutung der Unterstützung der Lernenden als ein zentrales Qualifikationsmerkmal von Online-Angeboten und deren Realisierung in SOMEK vorgestellt. Brüggen stellt in seinem Artikel fest, dass die hohen Erwartungen die bisher mit dem Einsatz von eLearning verknüpft waren nicht erfüllt wurden, was er auf eine mangelnde Nutzerorientierung zurückführt. Vor diesem Hintergrund entfaltet Brüggen, mit Bezug zur konstruktivistischen und subjektorientierten Lerntheorie (Holzkamp), einen Ansatz, der die Lernerorientierung und deren Lerngründe als zentrale Kriterien bei der Umsetzung von eLearning-Angeboten ausweist. Heidenreich greift in ihrem Beitrag ebenfalls den Gedanken der Lernerorientierung auf und zeigt wie dieser im Projekt «SOMEK» umgesetzt wurde. Im Fokus steht dabei die Frage, wie Angebote möglichst passgenau und attraktiv für die anvisiere Lerngruppe umgesetzt werden kön- 
nen, was die Autorin anhand der Kriterien "Viability» und «Social Connectivity» veranschaulicht.

Der dritte Teil widmet sich der schwierigen, aber grundlegenden Thematik der Vernetzung und Kooperation. Asselmeyer hebt in seinem Beitrag hervor, dass die Kooperation und Vernetzung verschiedener Institutionen und Einrichtungen ein entscheidendes Kriterium darstellt, um Ressourcen effektiv zu nutzen und qualitativ hochwertige Angebote anbieten zu können. Er zeigt zudem, dass neben der Vertretung individueller Interessen, eine Kultur der Gemeinschaftlichkeit notwendig ist, um einen starke Verbundstruktur zu etablieren. Oppernowski und Rüggebrecht stellen in ihrem Beitrag dar, wie dieses Ziel im Modellprojekt umgesetzt wurde und diskutieren vor allem die organisatorischen Herausforderungen, die mit einem großen Verbund aus unterschiedlichen Institutionen hervorgehen. Sie betonen dabei u.a. die Notwendigkeit der Ausbildung einer Projektkultur und eines effizienten Kompetenzen- und Ressourcenmanagements.

Den Abschluss bildet ein Einblick in bereits realisierte Projekte im Bereich der Online-Vermittlung von Medienkompetenz, die den Band abrunden. Fritische und Pannerale stellen in inrem Beitrag das Online-Seminar «Erziehung zur Medienkompetenz» als Teil des bayrischen Erweiterungsstudiengangs «Medienpädagogik» vor, der von einem Verbund bayrischer Hochschulen angeboten wird. Interessant an dem Beitrag ist nicht nur die curriculare Ausgestaltung des Studiengangs, sondern auch die Darstellung der konkreten Erfahrung mit diesem, die belegen, wie wichtig die didaktische Gestaltung der Kurse und die Bereitstellung angemessener Werkzeuge zur Kommunikation und Kooperation sind. Paulmann stellt in seinem Beitrag das Online-Fortbildungsangebot «eL3» der Universitäten Erlangen-Nürnberg vor, bei dem besonderer Augenmerk auf die tutorielle Betreuung der Lernenden, im Rahmen der Sicherung von Nachhaltigkeit, gelegt wurde. Ebenso zeigt sich eine starke Fach- und Unterrichtsorientierung, als ein entscheidendes Kriterium für die ungewöhnlich hohe Akzeptanz des Online-Angebots. Aufenanger und Siller zeigen in ihrer Darstellung des Projekts «MeKoLLI» wie wichtig die Fall- und Problemorientierung bei der Vermittlung von Medienkompetenz für den Einsatz im Unterricht ist, ohne dabei die aktuellen Entwicklungen im Medienbereich als Hinter- 
grund für pädagogisches Handeln aus den Augen zu verlieren. Das von Frank vorgestellte «Leipziger Online-Seminar: Medienkompetenz» hebt sich von den bereits vorgestellten Projekten ab, da hier vor allem die Ausbildung von Online-Tutoren im Zentrum steht. Die theoretischen Bezüge des Projekts bilden die Projektmethode und ein auf dem Begriff der Medienkompetenz aufsetzende Begriff der eLearningkompetenz, der die Gestaltung und Umsetzung von Online-Seminaren umfasst.

Der Band «Mit eLearning zur Medienkompetenz» zeichnet sich vor allem durch seine Diversität, den Praxisbezug und die Integration verwandter Initiativen aus. Er verdeutlicht die Komplexität und die damit verbundenen Herausforderungen, die mit der Etablierung eines universitären onlinegestützten Weiterbildungsangebots im Bereich «Medienkompetenz» einhergehen. Diese reichen von der Ebene der technischen und didaktischen Gestaltung bis hin zur Etablierung und Strukturierung eines effektiv arbeitenden Verbunds. Besonders wertvoll sind hier die Konkretisierungen theoretischer Beiträge auf der Grundlage der Projekterfahrungen sowie die Einbeziehung bereits umgesetzter Projekte.

Insgesamt unterstreicht der Band zentrale Aspekte, die es bei der Etablierung von Online-Angeboten zu berücksichtigen gilt, die in der Praxis aber häufig vernachlässigt werden. Hierzu gehört zum einen die Erkenntnis, dass der Aufbau eines Projektverbunds kein Selbstläufer darstellt, sondern ein Prozess ist, den zu organisieren und zu koordinieren ein großes fachliches Know-How und vielfältige Ressourcen bedarf. Zentral ist auch die Feststellung, dass die Etablierung eines Online-Angebotes besonders im Kontext eines Verbundes und noch mehr in Perspektive auf die Veränderung des Klientels von Hochschulen, auch immer einen institutionellen Entwicklungsprozess umfasst, der u.a. die Frage nach dem Selbstverständnis und der Neuorganisation institutioneller Strukturen aufwirft. Darüber hinaus zeigen sich die didaktische Gestaltung, die Zielgruppen- und Berufsfeldorientierung sowie die nachhaltige Betreuung als zentrale Erfolgsfaktoren für Online-Angebote, die allerdings dem Gedanken einer kontextfreien Wiederverwertbarkeit von Angeboten tendenziell entgegenstehen. Für den Leser dieses Bandes wäre über diese facettenreiche Darstellung der konkreten Rahmenbedingungen hinaus noch eine Auseinandersetzung mit der grund- 
sätzlichen Frage nach den Potenzialen von eLearning-Angeboten für eine Förderung von Medienkompetenz hilfreich. So entfalten einige der Beiträge auch alternative Ansätze z.B. in Form von Blended-Learning-Konzepten, die neben der klassischen Vermittlung auch eine interessante Option darstellen. Insofern kann der Titel des Bandes auch als Frage verstanden werden: Mit eLearning zur Medienkompetenz? Zu Beantwortung dieser aktuellen Frage liefert der Band einen anregenden und differenzierten Beitrag, der viele der bei der Beantwortung dieser Frage zu berücksichtigenden Aspekte aufgreift und diese nicht nur theoretisch, sondern auch mit Bezug zur Praxis und konkreten Projekten diskutiert. In diesem Sinne stelle der Band einen aktuellen Beitrag zur Hochschulentwicklung im Zeichen der Wissensgesellschaft und des BolognaProzesse dar, der zudem einen relevanten Beitrag zur medienpädagogischen Diskussion liefert.

Alexander Unger 